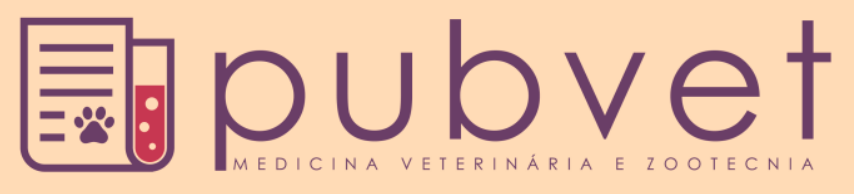

https://doi.org/10.31533/pubvet.v12n8a155.1-8

\title{
Hemimelia longitudinal pré-axial intercalar associada à hipoplasia umeral em cão: relato de caso
}

\author{
Clara Bastos da Rocha ${ }^{10}$

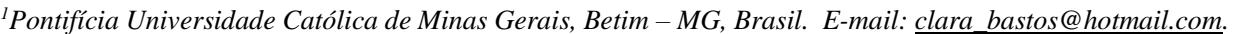

\begin{abstract}
RESUMO. Alterações na embriogênese podem produzir anomalias na estrutura ou função dos tecidos e órgãos e essas podem ser de origem genética ou ambiental. O processo conhecido como organogênese, dependerá da interação entre endoderma, mesoderma e ectoderma, que originará movimentos celulares posteriores, através da migração de células precursoras com a formação dos botões dos membros e assim acarretando na citodiferenciação, ou seja, na formação dos membros propriamente ditos. As disostoses, que são o conjunto de alterações morfológicas congênitas caracterizam-se pelo desenvolvimento anormal de um osso ou de parte dele, que pode acometer o esqueleto apendicular. A agenesia ou também conhecida como hemimelia, consiste em uma anomalia de caráter raro na qual, um ou mais ossos pareados possuem ausência completa ou parcial, mas dentre essa raridade a de maior frequência é a hemimelia na forma unilateral. O relato de caso trata-se de um cão, sem raça definida, apresentando deformidade de membro torácico esquerdo, claudicação e sem conseguir apoiá-lo ao chão. Foram realizados exames radiográficos do membro consecutivamente, e que revelaram a presença de hemimelia longitudinal pré-axial intercalar associada à hipoplasia umeral. Optou-se por aguardar o desenvolvimento do animal para reavaliações clínicas e desenvolvimento de prótese no membro acometido.
\end{abstract}

Palavras-chave: citodiferenciação, disostoses, embriogênese, hemimelia, hipoplasia umeral

\section{Pre-axial longitudinal hemimelia associated with humeral hypoplasia in dog: case report}

ABSTRACT. Changes in embryogenesis may produce abnormalities in the structure or function of tissues and organs and these may be of genetic or environmental origin. The process known as organogenesis will depend on the interaction between endoderm, mesoderm and ectoderm, which will originate subsequent cellular movements, through the migration of precursor cells with the formation of the limb buds and thus causing cytodifferentiation, that is, in the formation of the limbs themselves. The disostoses, which are the set of congenital morphological alterations, are characterized by the abnormal development of a bone or part of it, which can affect the appendicular skeleton. Agenesis, or also known as hemimelia, consists of a rare character anomaly in which one or more paired bones have complete or partial absence, but among this rarity the most frequent is hemimelia in the unilateral form. The case report is a dog, SRD, presenting a left thoracic limb deformity, limping and unable to support it to the ground. Radiographic examinations of the limb were performed consecutively, which revealed the presence of pre-axial longitudinal hemimelia associated with humeral hypoplasia. It was decided to wait for the development of the animal for clinical reassessment and development of prosthesis in the affected limb.

Keywords: citdifferentiation, disostoses, embryogenesis, hemimelia, humeral hypoplasia 


\title{
Hemimelia longitudinal pre-axial intercalar asociada a la hipoplasia humeral en perro: reporte de un caso
}

\begin{abstract}
RESUMEN. Los cambios en la embriogénesis pueden producir anomalías en la estructura o función de los tejidos y órganos y éstas pueden ser de origen genético o ambiental. El proceso conocido como organogénesis, dependerá de la interacción entre endoderma, mesoderma y ectoderma, que originará movimientos celulares posteriores, a través de la migración de células precursoras con la formación de los botones de los miembros y así acarreando en la citodiferenciación, o sea, en la formación de los miembros propiamente dichos. Las disostosis, que son el conjunto de alteraciones morfológicas congénitas, se caracterizan por el desarrollo anormal de un hueso o de parte de él, que puede acometer el esqueleto apendicular. La agenesia o también conocida como hemimelia, consiste en una anomalía de carácter raro en la cual uno o más huesos pareados poseen ausencia completa o parcial, pero de esa rareza la de mayor frecuencia es la hemimelia en la forma unilateral. El relato de caso se trata de un perro criollo, presentando deformidad de miembro torácico izquierdo, claudicación y sin conseguir apoyarlo al suelo. Se realizaron exámenes radiográficos del miembro consecutivamente, y que revelaron la presencia de hemielia longitudinal pre-axial intercalar asociada a la hipoplasia humeral. Se optó por aguardar el desarrollo del animal para reevaluaciones clínicas y desarrollo de prótesis en el miembro afectado.
\end{abstract}

Palabras clave: citodiferenciación, disostosis, embriogénesis, hemimelia, hipoplasia humeral

\section{Introdução}

Alterações na embriogênese produzem anomalias na estrutura/função dos tecidos e os defeitos congênitos podem ser de origem genética e/ou ambiental (Corbera et al., 2002; Alam et al., 2006; Kasinger et al., 2014; Bezerra et al., 2016). $\mathrm{Na}$ gestação, os membros dos vertebrados e outros animais são originados pela organogênese, que consiste na formação dos rudimentos de vários órgãos que compõem o corpo. Esse é dividido em três distintas fases: pré-implantação, 0 a 16 dias; embriogênese, 16 a 30 dias e crescimento fetal, do $30^{\circ}$ dia até o final da gestação (Filadelpho \& Souza, 2009). Tal processo dependerá da interação entre as três camadas germinativas: endoderma, mesoderma e ectoderma que envolve movimentos celulares posteriores. $\mathrm{O}$ mesoderma, se divide em placa lateral do mesoderma somático e somitos, sendo a primeira fonte exclusiva de precursores esqueléticos e o segundo de precursores musculares para toda a formação estrutural do animal (Ingrith et al., 2002; Breuer \& Towle, 2004).

Nele ocorrem não só a citodiferenciação, mas também a morte programada de células, que é essencial para o formato dos membros e dígitos. Assim, células durante algum momento dos estágios de gástrula inicial e tardia são determinadas, promovendo o período crítico do desenvolvimento, que se dá por uma fase na qual determinado tecido ou órgão em desenvolvimento é mais sensível a determinados fatores (Inghith et al., 2002; Filadelpho \& Souza, 2009).

Em cães, ess período ocorre a partir do $22^{\circ}$ ao $35^{\circ}$ dia gestação (Ingrith et al., 2002; Alam et al., 2006; Alievi et al., 2012; Campos et al., 2013; Lanteri et al., 2014; Dona et al., 2016), e em gatos varia desde o $16^{\circ}$ ao $28^{\circ}$ dia. Geralmente é neste estágio que os tecidos são mais sensíveis às anomalias do desenvolvimento. Passado esse período, as estruturas esqueléticas só tendem a aumentar de tamanho e manifestar suas características morfológicas maduras (Breuer \& Towle, 2004).

\section{Disostoses}

As disostoses são um conjunto de alterações morfológicas congênitas, caracterizadas pelo desenvolvimento anormal de um osso ou por parte dele (Breuer \& Towle, 2004; Alievi et al., 2012; Cinti et al., 2012; Lanteri et al., 2014; Costa et al., 2016), decorrentes de erros durante o desenvolvimento embrionário (Dona et al., 2016). É de caráter raro em cães e gatos (Alam et al., 2006; Mckee \& Reynolds, 2007; Alievi et al, 2012), mas apresenta maior prevalência em felinos. São alterações pouco diagnosticadas e quando diagnosticadas, não são relatadas levando a dificuldade de avaliação da incidência real em cães e gatos (Breuer \& Towle, 2004). Essas alterações do esqueleto apendicular podem ter diversas classificações, como: afalangia $(\mathrm{a}=\mathrm{sem}$, 
falange $=$ falange - ausência de um dígito ou de vários), ectrodactilia (ektroma $=$ aberto formação de fendas digitais congênitas que se estende entre os metacarpos), braquidactilia (brachus $=$ 'short' $=$ pequeno - tamanho reduzido dos dedos), polidactilia (polys = muitos, dactylos = dígitos - aumento do número de dígitos), sindactilia $($ syn $=$ juntos - dígitos adjacentes são fundidos) (Breuer \& Towle, 2004; Dona et al., 2016).

\section{Hemimelia}

A hemimelia, também conhecida como agenesia ou hipoplasia de ossos pareados (Mckee \& Reynolds, 2007; De Paula \& Filgueira, 2012), consiste em uma patologia que na qual os ossos de conformação paralela são parcial ou totalmente ausentes, e pode se apresentar de forma unilateral ou bilateral, sendo unilateral de maior frequência (Alam et al., 2006; Alievi et al., 2012; Cinti et al., 2012; Campos et al., 2013; Bezerra et al., 2016; Costa et al., 2016; Kene et al., 2017).

Ela pode ser classificada como terminal, quando há ausência de parte ou totalidade dos ossos distais, ou intercalar, quando ocorre em osso intermediário (Figura 1) (Breuer \& Towle, 2004; Alievi et al., 2012; Cinti et al., 2012; Kene et al. 2017).

Ambas podem ser chamadas de transversas, quando um ou mais ossos estão ausentes no aspecto transversal do membro, ou longitudinais, quando estão ausentes ao longo do lado lateral ou medial do membro (Figura 1) (Alam et al., 2006; Alievi et al., 2012; Cinti et al., 2012; Campos et al., 2013; Kene et al., 2017). E ainda, no caso de ossos pareados, como por exemplo, o rádio e ulna, elas se classificam como pré-axial e pós-axial, onde pré-axial há ausência do osso medial e pósaxial ausência do osso lateral (Figura 1) (Breuer \& Towle, 2004; Alam et al., 2006; Alievi et al., 2012; Campos et al., 2013). O rádio, a tíbia e ulna são as estruturas de maior comprometimento e quando há o envolvimento do rádio, os pacientes tendem a apresentar a sintomatologia da doença já nas primeiras semanas de vida (De Paula \& Filgueira, $\underline{2012)}$

\section{Etiopatogenia da Hemimelia}

A etiologia desta anomalia é incerta, existem fatores correlacionados entre genética, pelo acometimento em mais de um animal por ninhada ou com proximidades de parentescos e por ser considerada uma herança autossômica dominante, ambiental como radiações principalmente ionizantes (Alam et al., 2006; Kasinger et al., 2014). Podem ocorrer também devido a transtornos físicos, pois durante a gestação há uma séria deficiência de minerais como cobre, manganês e zinco, que é passada pela progenitora, e, podem haver também compressões fetais, vacinações e drogas ministradas (Corbera et al., 2002; Alam et al., 2006; Alievi et al., 2012; Cinti et al., 2012; Bezerra et al., 2016; Costa et al., 2016; Kene et al., 2017).

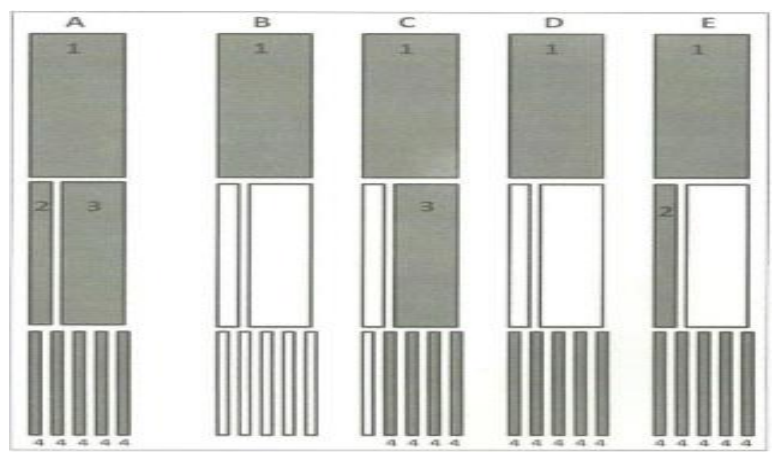

Figura 1. Classificações das hemimelias válida para qualquer osso do esqueleto apendicular. Exemplo de membro torácico. (1) úmero, (2) ulna, (3) rádio, (4) metacarpianos

(A) - Desenvolvimento normal dos ossos,

(B) - Hemimelia terminal transversa,

(C) - Hemimelia terminal longitudinal,

(D) - Hemimelia intercalar transversa,

(E) - Hemimelia intercalar longitudinal.

Fonte: Alievi et al., 2012.

Outra possível causa seria os defeitos vasculares, onde a vasculogênese (formação dos vasos na vida embrionária) precede normalmente a condrogênese (formação de cartilagem) e mielogênese (formação da medula óssea), sugerindo que uma vasculogênese anormal poderia resultar em hemimelia (Alam et al., 2006; Cinti et al., 2012; De Paula \& Filgueira, 2012; Campos et al., 2013).

Desta forma, pelo grau hereditário possivelmente característico da doença, é indicada a esterilização dos animais acometidos (Alievi et al., 2012; Almeida et al., 2012; De Paula \& Filgueira, 2012), tanto dos pais quanto dos filhotes, fêmeas e machos (Alievi et al., 2012). As malformações se manifestam ao nascimento, mas, também podem ser percebidas logo após o desenvolvimento do indivíduo (Costa et al., 2016). Quando ocorrem nas extremidades dos membros ou em partes deles, ou seja, no esqueleto apendicular, suas manifestações variam desde a ausência de uma única estrutura até ausência parcial ou completa do mesmo (Kasinger et al., 2014). 
Quando abordamos sobre a Hemimelia radial, nas primeiras semanas de vida são encontrados sinais de deformidade angular, claudicações e diminuição significativa da amplitude de movimento das articulações adjacentes (Cinti et al., 2012; De Paula \& Filgueira, 2012; Bezerra et al., 2016; Kene et al., 2017). Também contraturas dos músculos flexores de característica permanente devido à deformidade em varus e flexão do cotovelo e carpo (Breuer \& Towle, 2004; Mckee \& Reynolds, 2007; Cinti et al., 2012; Kene et al., 2017), arqueamento caudal da ulna e deformidades das articulações interfângicas, levando a disfunção do membro. Serão alterações sem manifestações dolorosas inicialmente, pelo fato de que o animal ainda é pequeno, leve e ainda não apresenta estímulos para uma deambulação adequada.

Em casos unilaterais, durante a deambulação o animal utiliza o lado lateral do membro e o carpo como pontos de sustentação, o que pode provocar ulcerações, tornando essa forma de sustentação incompatível com a vida em decorrência das seguidas infecções cutâneas (Alievi et al., 2012).

\section{Diagnóstico}

Como diagnóstico definitivo é crucial a realização de uma anamnese detalhada, exame clínico e físico do paciente, abordando um histórico indicando a idade em que a alteração se tornou visível, se houveram outros animais da mesma ninhada apresentando sintomatologia ou qualquer tipo de acometimento (Breuer \& Towle, 2004). Também é fundamental a realização de exames radiográficos (Cinti et al., 2012; De Paula \& Filgueira, 2012), mostrando que as partes ósseas podem apresentar tamanhos variados, maiores que o normal, no caso da ulna quando há ausência do rádio, ela se torna o único osso de sustentação, levando a um aumento de seu comprimento e encurvamento, acarretando possivelmente em luxação ou subluxação (Campos et al., 2013). Também permite observar ossos deformados ou ausentes, diagnosticando tanto casos de agenesia parcial quanto completa, e luxações de articulações (Bezerra et al., 2016) como a do cotovelo, devido ao membro se mostrar significativamente menor que o normal (Alievi et al., 2012), além do que também detecta a ausência do osso acometido e desvios angulares, avaliando articulações proximais e distais, considerando que em alguns casos se faz necessária a avaliação sob tomografia computadorizada para planejamento cirúrgico.

Portanto com um diagnóstico precoce, haverá a escolha da melhor terapia a ser realizada no paciente, tornando o tratamento mais adequado e tenderá a um possível prognóstico reservado a favorável (Alievi et al., 2012).

\section{Tratamento}

O tratamento conservativo ou cirúrgico variará de acordo com a localização da agenesia (Breuer \& Towle, 2004) e a abordagem de cada um deles dependerá da função do membro e o grau de deformidade (Breuer \& Towle, 2004; Cinti et al., 2012). Dos tratamentos conservativos são descritos a bandagem de Robert Jones em posição de apoio, talas que levarão a formação de uma anquilose óssea acompanhada por exames radiográficos, (Breuer \& Towle, 2004; Khotwal et al., 2011) próteses e órteses (Almeida et al., 2012). Os tratamentos cirúrgicos serão reconstrução do membro com enxertos ósseos, artrodeses, osteogênese pelo método de Ilizarov, onicectomia e, além da possibilidade da amputação do membro acometido (Breuer \& Towle, 2004; Alievi et al., 2012; Campos et al., 2013).

\section{Prognóstico}

O prognóstico do caso, para retorno à função do membro, sem cirurgia geralmente é desfavorável e há até a sugestão de eutanásia em casos bilaterais. No entanto, os animais com essas condições quando tratados de forma conservativa costumam apresentar boa qualidade de vida, portanto, o tratamento parece ser eticamente justificável, principalmente se a eutanásia for alternativa (Breuer \& Towle, 2004).

\section{Relato de caso}

Foi resgatada por uma clínica veterinária na cidade de Belo Horizonte - MG uma fêmea, da espécie canina, SRD, de aproximadamente dois anos de idade (análise pela arcada dentária). Pela ausência de histórico detalhado da paciente, realizou-se imediatamente exames clínicos e físicos, onde notou-se mucosas levemente hipocrômicas, abdômen evidentemente distendido, moderada dor a palpação, temperatura retal de $39,5^{\circ} \mathrm{C}$, prostrada, não estava se alimentando bem, apresentando os mamilos bem dilatados e sensíveis ao toque. Além disso, corrimentos vaginais de aspecto sanguinolento. Pela hipótese de uma possível gestação realizou- 
se ultrassonografia abdominal primeiramente, visto que, não sabendo o tempo exato de gestação, ele é considerado o método mais indicado quanto a seguridade dos filhotes. Com ele foi possível obter a contagem do número de fetos, observação direta da atividade do coração e suas movimentações, possibilitando assim, a avaliação da viabilidade dos filhotes. E a partir da medida do raio de seus crânios estimou-se a idade gestacional de cinquenta e cinco dias.

Para melhor análise de seus parâmetros hematológicos, foram coletados da mãe exames de sangue para perfil check up de emergência (hemograma completo, TGP, perfil renal e urina), pelo fato de que em alguns dias a paciente estará entrando em trabalho de parto e analisar se em todo esse tempo houve algum acometimento direto com os filhotes. Também, exames sorológicos para detecção de Leishmania infantum (RIFI, ELISA e teste de triagem DPP ${ }^{\circ}$ LVC), visto ser uma cadela de rua, potencialmente imunossuprimida e sem qualquer proteção à contaminação. Felizmente a sorologia apresentou resultado negativo para Leishmania infantum e dentre seus parâmetros hematológicos observouse uma leve anemia, proveniente possivelmente de sua potencial desnutrição. Assim, iniciou-se imediatamente suplementação nutricional com polivitamínicos e cálcio.

No dia 26/04/2016, cinco dias após o resgate, por volta das $9 \mathrm{~h}$, a cadela iniciou trabalho de parto, finalizando às $14 \mathrm{~h}$ com nascimento de oito filhotes, duas fêmeas e seis machos. Quando avaliado os filhotes, um dos machos natimorto, apresentou anencefalia e apenas dois dígitos em MPD. O outro, aos dois dias de vida apresentou alteração anatômica de membro torácico esquerdo que foi observado através de exames clínicos e físicos. Com 45 dias de vida, houve certa dificuldade na manipulação do membro acometido para realização de exames de imagens, diagnosticando de agenesia de rádio e ulna (Figura 2), pelo fato de que o paciente apresentou desenvolvimento adequado da escápula e em posição anatômica e o osso que entendíamos como o úmero em posição diferente do normal e que nos levou um diagnóstico equivocado sobre a ausência completa dos ossos rádio e ulna.

Visto a alteração anatômica desse filhote, foram realizados alguns estudos radiográficos em seu membro (MTE, em posição LLD), para acompanhamento e avaliação do desenvolvimento ósseo da região acometida.

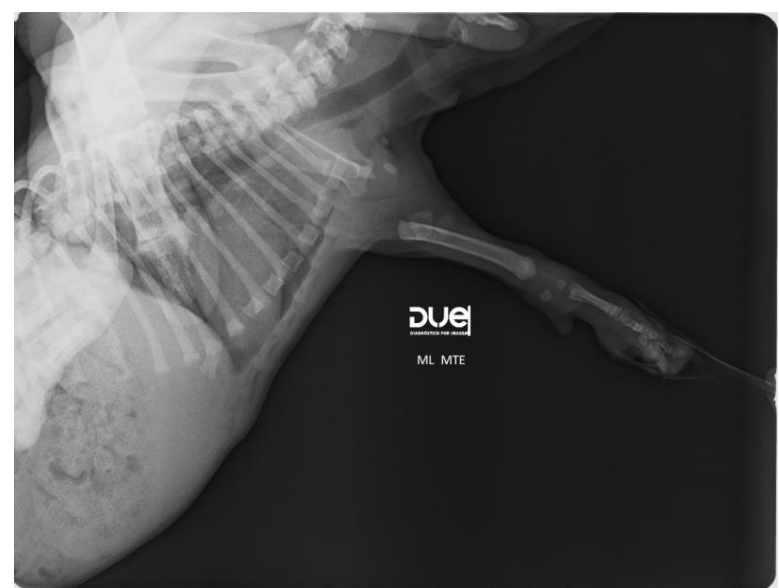

Figura 2. Primeira radiografia digital realizada no paciente com alteração anatômica evidente, aos 45 dias de vida, em posição ML de MTE. Obteve-se certa dificuldade quanto ao diagnóstico inicial de agenesia de rádio e ulna, pela dificuldade de manipulação do membro acometido para realização do exame de imagem. Foi avaliado a presença da escápula em boa conformação, úmero em posição diferente do normal, o que levou ao entendimento de ausência completa de rádio e ulna.

Atualmente, com 1 ano de idade, seu diagnóstico é reconhecido como Hemimelia longitudinal pré-axial intercalar associada à hipoplasia umeral, uma vez que o paciente apresentou a escápula em posição anatômica e boa conformação, na formação do úmero nota-se a presença de alguns fragmentos que se acredita serem áreas de metáfise proximal e distal do úmero, sendo classificado como hipoplasia umeral. Na região de rádio e ulna, observa-se a ausência total de rádio, classificando-a como hemimelia longitudinal pré-axial intercalar (Figura 3).

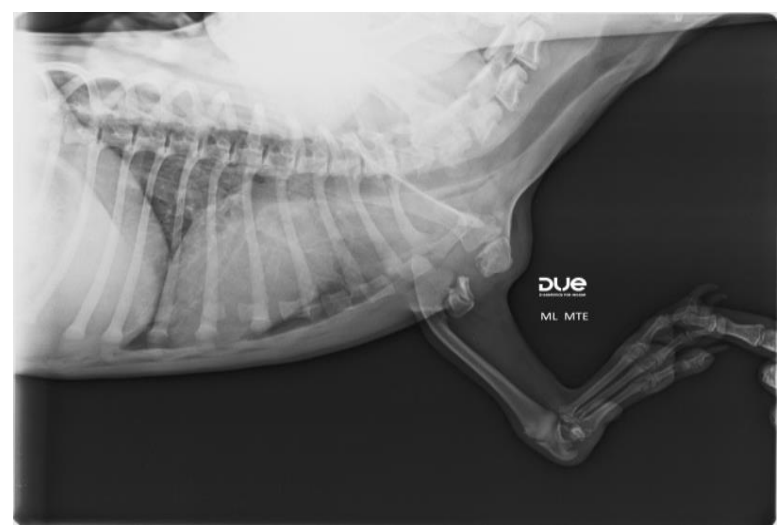

Figura 3. Alguns exames radiográficos digitais foram realizados afim de monitorar o crescimento ósseo da paciente em desenvolvimento, visto a dificuldade de diagnóstico e manipulação do membro acometido aos 45 dias de vida. Aos três meses de vida, evidenciou em Posição ML de TEM, escápula em ótima conformação e em posição anatômica, áreas de metáfise proximal e distal do úmero, ulna em ótimo desenvolvimento e ausência completa do rádio. Malformação denominada de Hemimelia longitudinal pré-axial intercalar associada à hipoplasia umeral. 
$\mathrm{O}$ efeito de constantes terapias como acupunturas e fisioterapias tem auxiliado no desenvolvimento adequado do restante do membro e musculatura adjacente. Por tal motivo, não se optou pela amputação alta do MTE pelo notável desenvolvimento e adaptações posturais diante da anomalia.

O paciente não apresenta dificuldades para realizar suas atividades diárias e necessidades fisiológicas, porém, sabe-se que a ausência de um membro torácico ocasiona sobrecarga compensatória em membro torácico contralateral ocasionando, a médio prazo, desvio angular das articulações, bem como processos de degeneração articular precoce.

No intuito de minimizar as alterações compensatórias pelo desvio de marcha apresentado pelos pacientes com três membros pretende-se desenvolver uma prótese (Figura 4).

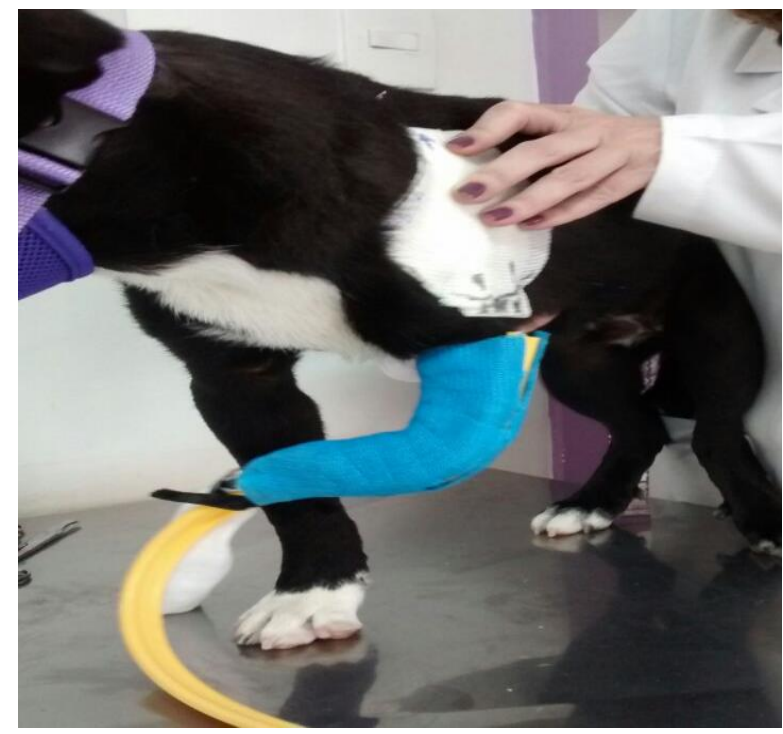

Figura 4. Primeiro molde de prótese realizado em paciente, apresentando a hipótese de diminuir as alterações compensatórias provenientes do desvio de marcha, visto que a ausência do membro levará a uma sobrecarga compensatória do membro contralateral e que a médio prazo, acarretará em desvios angulares e possíveis processos de degeneração articular.

\section{Discussão}

De acordo com De Paula \& Filgueira (2012), a má formação congênita tem maior ocorrência em membros torácicos do que pélvicos, uma vez que os membros torácicos se desenvolvem mais cedo e Alam et al. (2006), citam que as estruturas ósseas crescem de proximal para distal, portanto, independente do desenvolvimento anormal de determinada estrutura, pode ocorrer o desenvolvimento da estrutura distal sem qualquer tipo de alteração. $\mathrm{O}$ caso corrobora com a literatura, uma vez que, o membro torácico foi acometido apresentando a escápula em posição anatômica e boa conformação, áreas de metáfise próxima e distal de úmero, ausência total do rádio e ulna em perfeita conformação.

A etiologia desta malformação é incerta, existindo fatores correlacionados entre genética, pelo acometimento em mais de um animal por ninhada ou por proximidades de parentesco e também, pode ser considerada uma herança autossômica dominante (Corbera et al., 2002; Alam et al., 2006; Alievi et al., 2012; Bezerra et al., 2016; Costa et al., 2016). Além disso, fatores físicos, pelo fato de que durante o parto há uma séria deficiência de minerais como: cobre, manganês e zinco pela deficiência nutricional passada pela mãe e também compressão fetal, vacinações e drogas ministradas.

Em vista do caso, pelo fato de ter sido uma cadela resgatada, não se sabe seu verdadeiro histórico, então, uma das grandes suspeitas do acometimento dos filhotes seria talvez físico pela desnutrição vinda da mãe, anemia leve mostrada em seus exames hematológicos e a própria compressão fetal pelo número de filhotes e por não saber se houveram vacinações e drogas ministradas anteriormente na fêmea, durante o período de 22 a 35 dias de gestação. A genética também seria uma forte suspeita, devido a pelo menos mais um na ninhada ter aparecido com um tipo de malformação.

De acordo com De Paula \& Filgueira (2012), quando as estruturas acometidas são o rádio, a tíbia ou a ulna, que são geralmente as de maiores acometimentos, os pacientes tendem a apresentar sintomatologia da doença já nas primeiras semanas de vida. E também, nas primeiras semanas de vida, são encontrados sinais de deformidade angular, claudicações e diminuição significativa da amplitude de movimento das articulações adjacentes (De Paula \& Filgueira, 2012; Bezerra et al., 2016). O caso clínico se relaciona com a literatura, pelo fato de a malformação ter sido potencialmente no rádio e no úmero e o paciente apresentou a sintomatologia referente aos achados, como: desvio angular das articulações, claudicações e diminuição da amplitude de movimento.

De Paula \& Filgueira (2012), também afirmaram que é fundamental a utilização de exames radiográficos, mostrando que as partes 
ósseas podem apresentar tamanhos variados, maiores que o normal, no caso da ulna quando há ausência do rádio, ela se torna o único osso de sustentação, levando a um aumento de seu comprimento e encurvamento (Campos et al., 2013). O paciente passou por alguns exames de imagem durante todo o seu desenvolvimento ósseo, mostrando com evidência a posição anatômica correta da ulna e ausência completa do rádio, sendo o único osso de sustentação, levemente mais alongado que o normal.

O tratamento varia de acordo com a localização da deformidade (Breuer \& Towle, 2004) e de acordo com Breuer \& Towle (2004), Campos et al. (2013) e Bezerra et al. (2016), indicam a utilização da bandagem de Robert Jones em posição de apoio. Em vista do caso, o primeiro procedimento feito com o paciente foi a utilização da bandagem almofadada de Robert Jones, que é o método conservativo que manteve a posição de apoio do filhote até que houvesse o término do crescimento ósseo de seu membro.

De acordo com Breuer e Towle (2004), quando os animais com essas condições quando tratados de forma conservativa, costumam apresentar boa qualidade de vida, portanto, o tratamento parece ser eticamente justificável. Hoje, o paciente se comporta muito bem, obteve o tratamento conservativo assim que apresentado a deformidade angular do membro acometido, onde auxiliou-o no desenvolvimento e na deambulação normal, evitando que houvesse agravamentos na funcionalidade do membro contralateral, e apresenta uma vida completamente normal atualmente com 1 ano de idade, se movimentando e realizando suas necessidades fisiológicas.

Almeida et al. (2012) falaram sobre utilizações temporárias de próteses e órteses. Dentre o caso abordado, houve a hipótese da utilização de uma prótese para o paciente, o qual traria o conforto e estabilidade necessários para o paciente apresentar melhor qualidade de vida. Essa hipótese ainda está em andamento.

\section{Considerações finais}

Ao realizado esse relato, foi possível observar o quão importante é o diagnóstico desta malformação, visto que é uma anomalia de caráter extremamente raro em animais.

A medida que a medicina juntamente com a medicina veterinária avançam, os tratamentos evoluem juntamente a elas, assim, foi observado no presente estudo o crescente número de tratamentos tanto conservativos quanto cirúrgicos que podem ser introduzidos como tratamentos para tais malformações, mostrando ainda que, os conservativos quando associados a métodos complementares como fisioterapias e acupunturas, bandagens e também órteses e próteses, podem trazer uma melhor qualidade de vida ao paciente, promovendo o máximo possível da deambulação normal, sem a necessidade da hipótese de amputação do membro acometido, que foi considerada por anos como escolha de método terapêutico.

As malformações tem sido um assunto de estudo que se desenvolve cada dia mais, mas, ainda há necessidade de maiores relatos e análises minuciosas quanto a origem delas e a possibilidade novas formas de tratamento.

\section{Referências Bibliográficas}

Alam, M. R., Heo, S. Y., Lee, H. B, Kim, J. H., Park, Y. J., Lee, K. C., Choi, I. H. \& Kim, N. S. 2006. Preaxial longitudinal intercalary radial Hemimelia in a dog: a case report. Journal Veterinarni Medicina, 51(3), 118-123.

Alievi, M. M., Ferreira, M. P., Nóbrega, F. S., Gonzalez, P. C., Dai-Bó, I. S. \& Beck, C. A. C. 2012. Agenesia bilateral de rádio em felino (Felis Catus Domesticus) - relato de caso. Revista Clínica Veterinária, 97, 36-40.

Almeida, P. G., D'ávila, G. L., Scherer, S., Kunzler, K.C., Alievi, M. K. \& Streda, E. 2012. Agenesia de metacarpo, metatarso e falange em dois cães - Relato de caso. Archives of Veterinary Science, 17 (supl.), Resumo 126.

Bezerra, K. S., Cruz, T. P. P. S., Makino, H., Lima, S. R., Travaglin, D. R.P., Nespoli, P. B. \& De Almeida, A.B.P.F. 2016. Hemimelia parcial unilateral de rádio em felino. Acta Scientiae Veterinariae, 44(0), 1-4.

Breuer, G. J. \& Towle, H. A. M. 2004. Dysostosis of the canine and feline appendicular skeleton. Journal of the American Veterinary Medical Association, 225(11), 1685-1692.

Campos, W. N. S., Peres, T. P. S., Ruiz, T., Meireles, Y. S., Néspoli, P. B. \& De Souza, R. 2013. Hemlimelia bilateral de rádio em canino - relato de caso. Revista Semina: Ciências Agrárias, 34(5), 2381-2386.

Cinti, F., Del Magno S., Pisoni L. \& Joechler M. 2012. Bilateral radial hemimelia and multiple malformations in a kitten - Case Report. 
Journal of Feline Medicine and Surgery, 14(8), 598-602.

Corbera, J. A., Morales, M., Pulido, M., Juste, M. C. \& Gutierrez, C. 2002. Radiological Findings in Three Cases of Paraxial Radial Hemimelia in Goats. Journal of Veterinary Medical Science, 64(9),843-845.

Costa, M. C., Medeiros, F. J. S., Moreira, T. A., Modesto, T. C., Serra, T. L. \& Tsuruta, S.A. 2016. Transverse terminal humeral bilateral hemimelia in a dog - case report. Acta Veterinaria Brasílica, 10(4),368-372.

De Paula, V. V. \& Filgueira, K. D. 2012. Hemimelia Radial em um exemplar da espécie felina. Archives of Veterinary Science, 17(86), 261-263.

Dona, F. D., Meomartino, L., Valle, G. D., Lamagna, F. \& Fatone, G. 2016. Congenital deformity of the distal extremities in three dogs. Open Veterinary Journal, 6(3), 228-233.

Filadelpho, A. L. \& Souza, C. C. 2009. Ectrodactilia em cães - Revisão de Literatura. Revista Científica Eletrônica de Medicina Veterinária, 7, Ano XII.

Ingrith, A. M., Martins, V. M., Stolf, L. \& Busato, C. M. 2002. Dimelia anterior esquerda incompleta em cães: Relato de caso. Revista de Ciências Agroveterinárias, 1(1).

Kasinger, S. A., Marchiori, M. O.; Schmith R. A., Janaina R. V., Kickhofel, L. A., Schuster, A. G., \& Curcio, B. R. 2014. Amelia de membros torácicos em potro árabe: relato de caso. Veterinária e Zootecnia, 21(2), 260-264.

Kene, R. O. C., Oviawe, E. I., Yakubu, A. S., Buhari, S. \& Mayaki, A. M. 2017. Radiographic finding of radial hemimelia in a 6-day-old west African dwarf goat with a fractured ulna. Austin Journal of Radiology, 4(1), 1-3.

Khotwal, P. P., Soral, A. \& Varshney, M. K. 2011. Comparison of surgical treatment and nonoperative management for radial longitudinal deficiency. The Journal of Hand Surgery (European Volume), 37(2), 161-169.

Lanteri, G., Macri, F., Rapisarda G., Pietro, D., Auriemma, E. \& Marino, F. 2014. Congenital absence of humerus with preaxial terminal longitudinal hemimelia and hypoplasia of the scapula in a dog: a case report. Journal Veterinarni Medicina, 59(10), 506-510.

Mckee, W. M. \& Reynolds, J. 2007. Ulnocarpal arthrodesis and limb lengthening for the management of radial agenesis in a dog. Journal of Small Animal Practice, 48, 591-595.

Recebido: 14 Jun. 2018.

Aprovado: 21 Jul. 2018

Publicado: 7 Ago. 2018

Licenciamento: Este artigo é publicado na modalidade Acesso Aberto sob a licença Creative Commons Atribuição 4.0 (CC-BY 4.0), a qual permite uso irrestrito, distribuição, reprodução em qualquer meio, desde que o autor e a fonte sejam devidamente creditados. 